\title{
Virtual chromoendoscopy can be a useful software tool in capsule endoscopy
}

\author{
Gabriela Duque, Nuno Almeida, Pedro Figueiredo, Pedro Monsanto, Sandra Lopes, Paulo Freire, \\ Manuela Ferreira, Rita Carvalho, Hermano Gouveia and Carlos Sofia
}

Department of Gastroenterology. Faculty of Medicine University of Coimbra. Coimbra University Hospital. Coimbra, Portugal

\begin{abstract}
Background: capsule endoscopy (CE) has revolutionized the study of small bowel. One major drawback of this technique is that we cannot interfere with image acquisition process. Therefore, the development of new software tools that could modify the images and increase both detection and diagnosis of small-bowel lesions would be very useful. The Flexible Spectral Imaging Color Enhancement (FICE) that allows for virtual chromoendoscopy is one of these software tools.

Aims: to evaluate the reproducibility and diagnostic accuracy of the FICE system in CE.

Methods: this prospective study involved 20 patients. First, four physicians interpreted 150 static FICE images and the overall agreement between them was determined using the Fleiss Kappa Test. Second, two experienced gastroenterologists, blinded to each other results, analyzed the complete 20 video streams. One interpreted conventional capsule videos and the other, the CE-FICE videos at setting 2. All findings were reported, regardless of their clinical value. Non-concordant findings between both interpretations were analyzed by a consensus panel of four gastroenterologists who reached a final result (positive or negative finding).

Results: in the first arm of the study the overall concordance between the four gastroenterologists was substantial (0.650). In the second arm, the conventional mode identified 75 findings and the CE-FICE mode 95. The CE-FICE mode did not miss any lesions identified by the conventional mode and allowed the identification of a higher number of angiodysplasias (35 vs 32), and erosions (41 vs. 24).

Conclusions: there is reproducibility for the interpretation of CE-FICE images between different observers experienced in conventional $\mathrm{CE}$. The use of virtual chromoendoscopy in $\mathrm{CE}$ seems to increase its diagnostic accuracy by highlighting small bowel erosions and angiodysplasias that weren't identified by the conventional mode.
\end{abstract}

Key words: Capsule endoscopy. Flexible Spectral Imaging Color Enhancement. Erosions. Angiodysplasias.

Received: 21-11-11.

Accepted: 02-03-12.

Correspondence: Gabriela Duque Department of Gastroenterology. Coimbra University Hospital. Avenida Bissaya Barreto. 3000-075 Coimbra, Portugal. e-mail: gabrieladuquepena@gmail.com
Duque G, Almeida N, Figueiredo P, Monsanto P, Lopes S, Freire $P$, Ferreira $M$, Carvalho $R$, Gouveia $H$, Sofia C. Virtual chromoendoscopy can be a useful software tool in capsule endoscopy. Rev Esp Enferm Dig 2012; 104: 231-236.

\section{INTRODUCTION}

Digestive endoscopy has evolved from a pure diagnostic technique into a major interventional and therapeutic one. Even though, its value as a diagnostic tool has been kept and multiple developments have occurred to increase its diagnostic accuracy. The current technology of imageenhanced endoscopy is available to augment the detection, diagnosis and treatment of subtle lesions (1). Conventional white light endoscopy is associated with a disproportionate miss rate for subtle lesions (mainly flat). Therefore, the endoscopic manufacturers have developed several adjunct technologies working as point enhancement. One of these software tools is the Flexible Spectral Imaging Color Enhancement (FICE). It is easier and less time consuming than topical application of stains or pigments, since it is available with a simple button change to make multiple modifications of wavelength (2).

The FICE system, manufactured by Fujinon Corporation (Saitama, Japan), is implemented based on Spectral Estimation Technology, which takes a real time endoscopic image from the video processor and arithmetically processes, estimates and produces an image of a given, dedicated wavelength of light for each of the three colors (red, green and blue). After changing the desired wavelengths with virtual electronic filters, the image is instantaneously reconstructed. This leads to the enhancement of tissue microvasculature as a result of the differential optical absorption of light by hemoglobin in the mucosa (2). The advantage of this new digital processing system is an enhancement in 
the detection and identification of pathologic changes and to diagnose clinical findings more accurately $(3,4)$.

Capsule endoscopy (CE) was developed to examine the small intestine and has become a well-established tool allowing direct visualization of the entire small bowel mucosa, although without the ability for tissue sampling and therapeutic option as in deep enteroscopy. Since it was introduced in the year 2000, its journey in such a short time and its impact on healthcare had been truly remarkable (5).

However, despite technical improvements in software, the diagnostic yield and quality of CE images are influenced by the speed of the video stream and, consequently by the time spent in the analysis of the recordings. The FICE software was very recently implemented within the software of the video capsule system (Rapid 6.0- Given Imaging Ltd. Yoqneam, Israel). During the analysis, the physician has the ability to modify the visualized video stream characteristics for optimal mucosal imaging by switching between standard and different FICE sets. Even though there aren't studies concerning this issue, recent published papers (6-8) suggest a potential role for FICE in CE.

The main aim of our study was to evaluate diagnostic accuracy of FICE system in CE and to determine if virtual chromoendoscopy can provide additional value over conventional capsule enteroscopy. Additionally we wanted to determine if the interpretation of CE-FICE images was reproducible between different gastroenterologists, experienced in conventional CE.

\section{PATIENTS AND METHODS}

We designed a prospective study, involving 20 consecutive CE recordings, performed in a University Hospital between August/2009 and November/2009. The indication for all capsule studies was obscure gastrointestinal bleeding (OGIB). All patients had been submitted to conventional endoscopic examination (upper digestive endoscopy and total colonoscopy) prior to CE. All gave their informed consent for the procedure.

A PillCam SB 2 (Given Imaging Ltd; Yoqneam, Israel) was used according to standardized protocol. Patients were submitted to a liquid diet on the eve of the exam. After an overnight fast of 12 hours, they presented to the ambulatory clinic for the ingestion of the capsule (with a small amount of water with simethicone) and for the placement of the recording device. No oral purge was administered. They were only allowed to ingest water and liquid yogurts, 2 and 4 hours after capsule ingestion, respectively. A moderate physical activity was advised and the sensor array and the recording device were removed around 8 to 9 hours after the beginning of the exam. The digital video image streams of the examinations were downloaded to the RAPID system.

The three spectral specifications of the FICE sets useful for CE are: FICE set 1 (wavelengths red $595 \mathrm{~nm}$, green 540 $\mathrm{nm}$, blue $535 \mathrm{~nm}$ ), set 2 (wavelengths red $420 \mathrm{~nm}$, green
$520 \mathrm{~nm}$, blue $530 \mathrm{~nm}$ ) and set 3 (wavelengths red $595 \mathrm{~nm}$, green $570 \mathrm{~nm}$, blue $415 \mathrm{~nm}$ ). For our study we arbitrarily selected set 2 since it seemed the one that better increased the contrast between the vascular network and the mucosal background.

In the first phase of our study one independent endoscopist selected one hundred and fifty static images from the CEFICE with or without abnormal findings. These images were then interpreted by four experienced CE gastroenterologists (each with more than $100 \mathrm{CE}$ examinations), blinded to each other results. They had previously interpreted at least $5 \mathrm{CE}$ examinations with FICE mode 2. The overall agreement between all them was achieved using the Fleiss Kappa Test.

Secondly, two gastroenterologists with the same ability of reading and with similar reading times (an interim quality control for our small bowel endoscopists revealed a significant agreement rate for all; results not published) interpreted independently the 20 digital video streams. One visualized the video using exclusively the CE conventional mode and the other one the FICE mode. They reviewed the videos at the same frame rate (10 per second) and were blinded to each other results. All findings were reported and individual lesions were classified as polyps, erosions, ulcers, sub-epithelial lesions, lymphangiectasia, angiodysplasia, phlebectasia, ulcerated stenosis and areas of mucosal atrophy. Findings detected only by one gastroenterologist (conventional mode $v s$. CE-FICE mode) were analyzed by the panel of four elements that participated in the first phase of the study. They decided, by consensus, if it was a real positive finding or not. Their final decision was assumed as the gold standard.

Inter-observer agreement between the four gastroenterologists was calculated using Fleiss Kappa coefficient. Kappa $<0.4$ was considered as poor agreement whereas between 0.41-0.6 as moderate, 0.61-0.80 substantial and 0.81-1.00 as excellent agreement. The differences between the number of findings for each mode were analyzed by McNemar's test; $p<0.05$ was considered statistically significant. Analysis was performed with Statistical Package for the Social Sciences (SPSS) v. 18.0.

\section{RESULTS}

We included 20 patients, 11 female and 9 male with an average age of $59 \pm 19.5$ years. A total of 20 videos, one for each patient was obtained. Visualization of the entire small bowel was possible in 95\% (19/20). Gastric transit time was $23,5 \pm 21,8 \mathrm{~min}(4-81)$ and for the 19 patients with total enteroscopy the small bowel transit time was $280,3 \pm 75,4$ $\min (190-479)$. There were no cases of capsule retention.

When assessing the inter-observer agreement between four gastroenterologists while assigning one hundred fifty static CE-FICE images using the Fleiss Kappa Test, the overall concordance was substantial $(0.650,95 \% \mathrm{CI}, 0.57$ to 0.73 ) (Table I). This determined that the interpretation CE-FICE images were reproducible between different observers. The fact that all the CE-experts were analyzing 
Table I. Evaluation of 150 capsule endoscopy Flexible Spectral Imaging Color Enhancement static images

\begin{tabular}{|c|c|c|c|c|c|}
\hline Images & Standard (n) & Observer 1 & Observer 2 & Observer 3 & Observer 4 \\
\hline Normal & 46 & 45 & 45 & 48 & 51 \\
\hline Angiodysplasia & 30 & 30 & 30 & 30 & 29 \\
\hline Erosions & 40 & 41 & 40 & 38 & 37 \\
\hline Ulcers & 13 & 13 & 14 & 13 & 13 \\
\hline Lymphangiectasias & 15 & 15 & 15 & 15 & 14 \\
\hline Polyps & 4 & 4 & 4 & 4 & 4 \\
\hline Sub-epithelial lesions & 2 & 2 & 2 & 2 & 2 \\
\hline
\end{tabular}

non-continuous static images should be emphasized because of its difficulty in establishing a diagnosis.

In the second part of our study the gastroenterologist dedicated to the conventional CE videos identified 75 different findings and the one with the CE-FICE mode setting 2 identified 95 (Table II). Twenty more findings were detected using the FICE mode, comparatively to the conventional mode. All these 20 findings were analyzed by the expert's consensus panel and confirmed as being positive ones. The conventional mode identified 75 findings and none of them was missed by CE-FICE. The most frequent findings were erosions (Figs. 1 and 2) and angiodysplasias (Figs. 3 and 4). The FICE mode identified 17 additional erosions, (41.5\%; $\mathrm{p}<0,001)$, and 3 additional angiodysplasias $(8.6 \% ; p=0,25)$. Indeed, the FICE mode allowed the identification of a superior number of erosions when compared to conventional mode by enhancing its inflammatory halo (Fig. 1). As stated before CE-FICE did not miss any gross lesions visualized by conventional $\mathrm{CE}$.

\section{DISCUSSION}

CE is, undoubtedly, the gold standard for endoscopic examination of the small bowel. Similarly to conventional endoscopy, modified imaging with enhanced vascular and mucosal contrast might improve detection and characteri-

Table II. Small bowel endoscopic findings (identified by the conventional mode, FICE mode and total) and variation considering both modes

\begin{tabular}{llll}
\hline Endoscopic findings & $\begin{array}{l}\text { Conventional } \\
\text { mode }\end{array}$ & FICE mode & Variability (n/\%) \\
\hline Erosions & 24 & 41 & $+17(41,5 \%)$ \\
Angiodysplasia & 32 & 35 & $+3(8,6 \%)$ \\
Polyps & 3 & 3 & 0 \\
Sub-epithelial lesions & 2 & 2 & 0 \\
Ulcerated stenosis & 1 & 1 & 0 \\
Other findings* & 13 & 13 & 0
\end{tabular}

*Other findings: lymphanglectasias and mucosal atrophy areas.

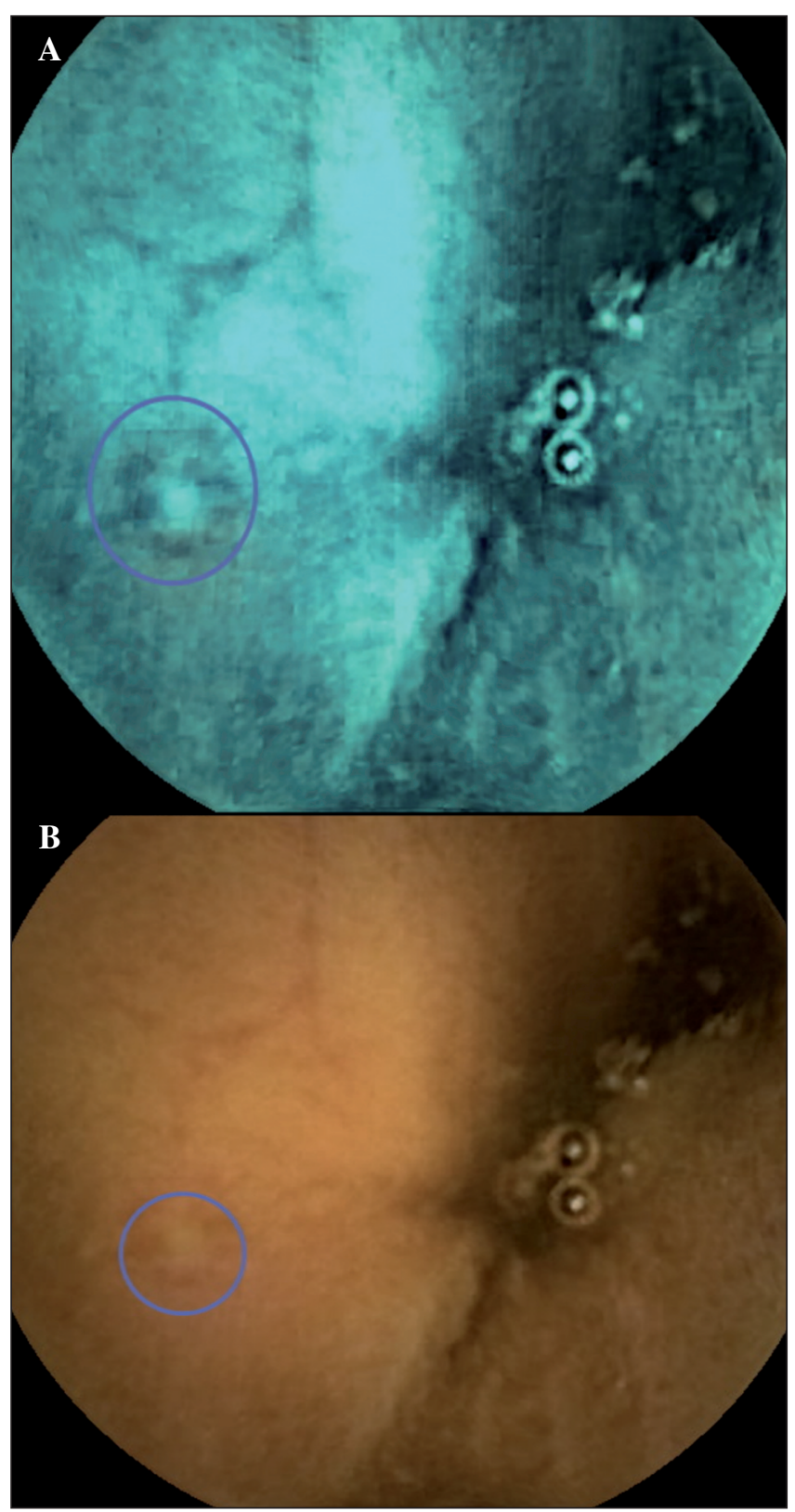

Fig. 1. Erosions identified with FICE mode (A) but not identified with conventional mode (B). 


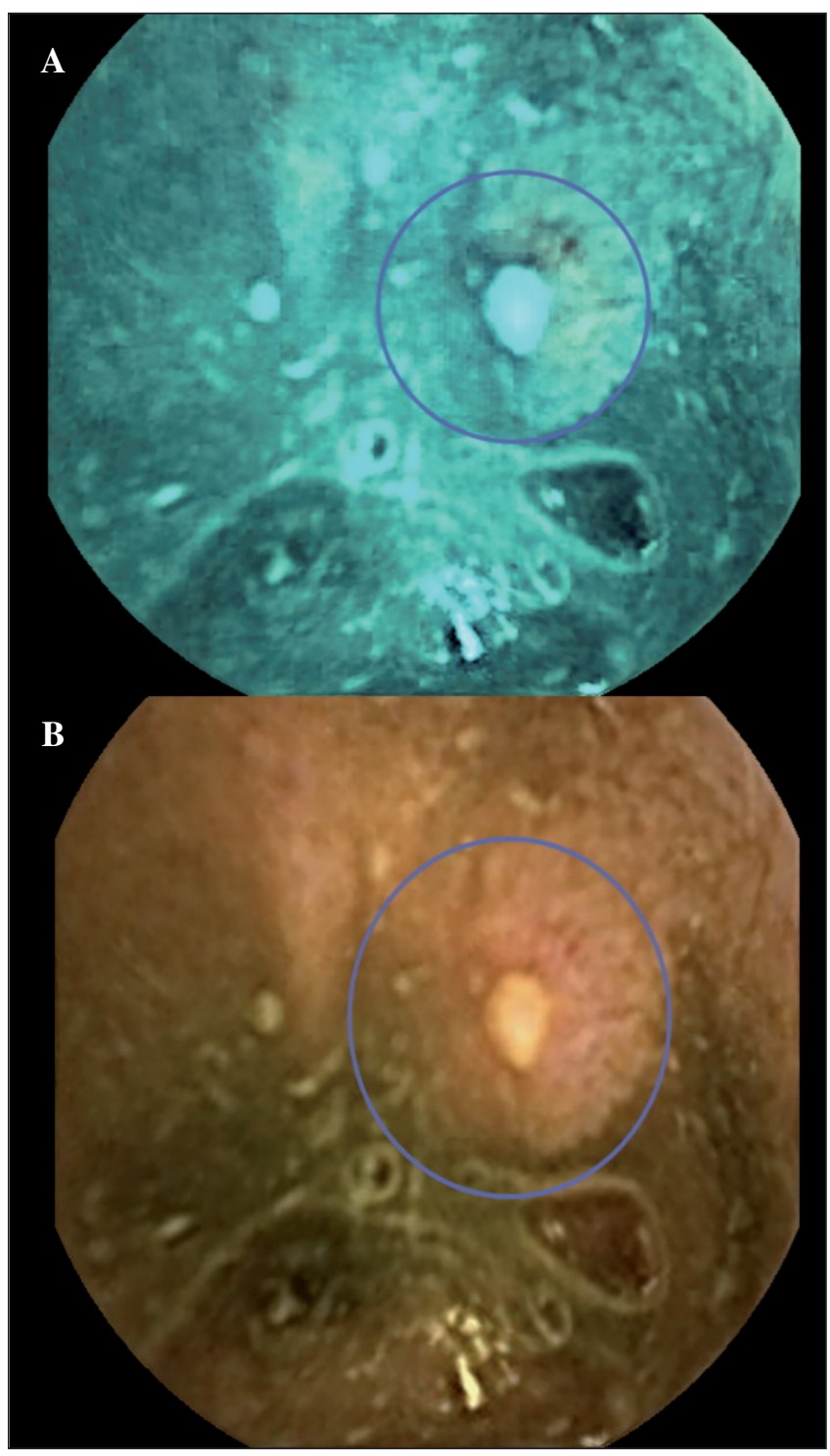

Fig. 2. Erosions identified with both modes.

zation of small-bowel lesions. FICE is a digital imaging technology that is executed by external software and allows the processing of regular images captured by standard devices. Nevertheless, there are major differences between the applications of FICE in flexible endoscopy and in CE. In fact, the resolution of the images is higher in real time endoscopy and it is possible to use flexible focus and magnification, techniques not available in CE (6).

The deferred way of viewing and scoring CE video leads to difficulties in observation and interpretation. Software designed to increase diagnostic accuracy of physicians is necessary and desired. FICE increases vascular contrast emphasizing lesion hypervascularity and vascular morphology, the inflammatory halo from the ulcers and erosions, the angiodysplasia, and other vascular abnormalities. FICE might also aid in differentiation of neoplastic/non-neoplastic

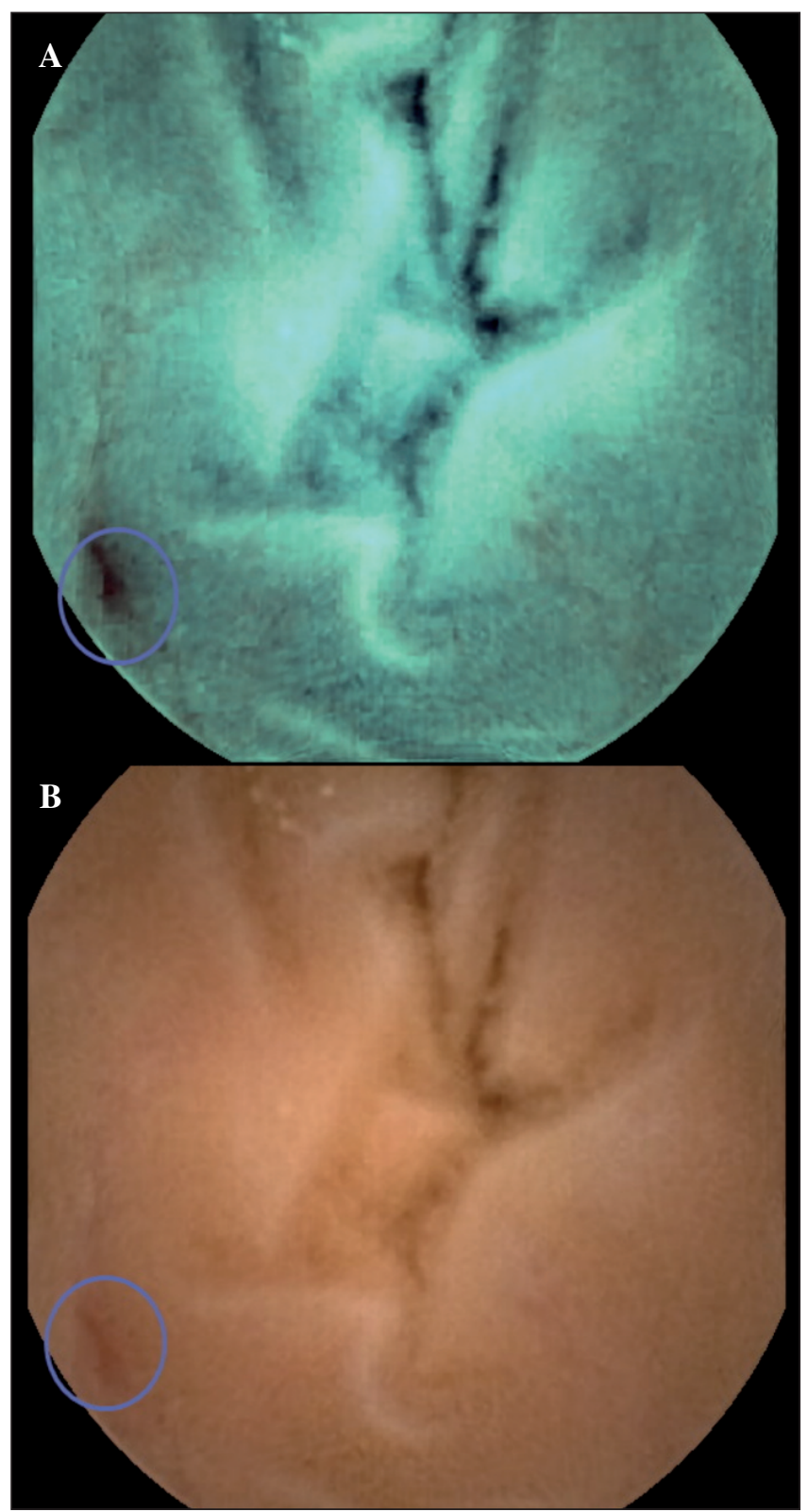

Fig. 3. Angiodysplasia identified with FICE mode (A) but not identified with conventional mode (B).

lesions in the small-bowel as it has been shown in upper endoscopy and colonoscopy $(1,4,9,10)$.

In the RAPID CE software there are three different FICE settings and it remains to be verified which of these would be the most appropriate. Sparse prior retrospective studies have determined the utility of FICE system in CE, analyzing isolated images. Pohl et al. assumed in their work that setting 1 achieved the preferred appearance (3) and Imagawa et al. (7) found that CE-FICE was useful, but only in settings 1 and 2. Prospective studies aiming to determine the potential utility of FICE virtual chromoendoscopy in small-bowel capsule enteroscopy are even fewer. Imagawa et al. (8), in a 
prospective study, again identified settings 1 and 2 as allowing better identification, mainly of angioectasias. In our case, setting 2 was chose and applied. In fact, when the study was designed there were no publications about the use of FICE in $\mathrm{CE}$ and no specific recommendations about the most useful setting in these specific cases. All above mentioned manuscripts were published after our work was completed and even now there is still no agreement concerning the specific setting to choose. We opted for the second one (red $420 \mathrm{~nm}$; green $520 \mathrm{~nm}$; blue $530 \mathrm{~nm}$ ) because it seemed the one that, in our initial experience, better increased the contrast between the vascular network and the mucosal background. It is known that penetration of light into the mucosa varies according to the wavelength: wavelengths between 400-500 nm are ideal for analyzing surface structures whereas longer wavelengths, of around $550 \mathrm{~nm}$, are more effective for visualizing blood vessels $(2,3)$. With this setting we planned to have a better surface mucosal structures observation and simultaneously a more effective identification of blood vessels. We did not use all different settings because we wanted to compare conventional $\mathrm{CE}$ with $\mathrm{CE}-\mathrm{FICE}$ in the context of daily clinical practice. The use of all settings would undoubtedly increase the reading time substantially and we think this is not feasible in the daily practice. Although we did not present the results of the reading times, we think these were no significantly different for both endoscopists. On the other side, if one observer visualized the same video in the three different settings an increase in diagnostic yield would be possible. Probably, the best way to compare the different settings is to have four different observers, with comparable diagnostic capabilities, one for each mode (conventional; CE-FICE settings 1, 2 and 3).

A limitation of our study was the small sample size and the relative inexperience of our team with CE-FICE. A previously quality control study (not published) revealed a substantial agreement for all in the conventional mode but there was no data for CE-FICE. So, the first phase of our study demonstrated reproducibility in the interpretation of CE-FICE images for the four gastroenterologists. With these results we confirmed that all involved elements had good diagnostic skills and any divergences detected would be determined by the technique (conventional CE vs. CEFICE) and not by differences in the endoscopists' skills. False positive findings were also eliminated since an expert panel reviewed all discordant results and a final consensus was deemed necessary to assume a positive finding.

Our work demonstrated an increased capability in diagnosing erosions in the FICE mode because of the enhancement of its inflammatory halo. The precise relevance of this finding is arguable, considering its doubtful clinical importance. Lacerations, erosions and ulcers might be present in 10 to $13.8 \%$ of healthy controls submitted to CE study (11). This percentage rises to $71 \%$ in patients under therapeutics with non-steroidal anti-inflammatory drugs (NSAIDs). The discrimination of these ulcerative lesions according to their etiology seems impossible to establish based on endoscopic images. Clinical application of CE in the management of

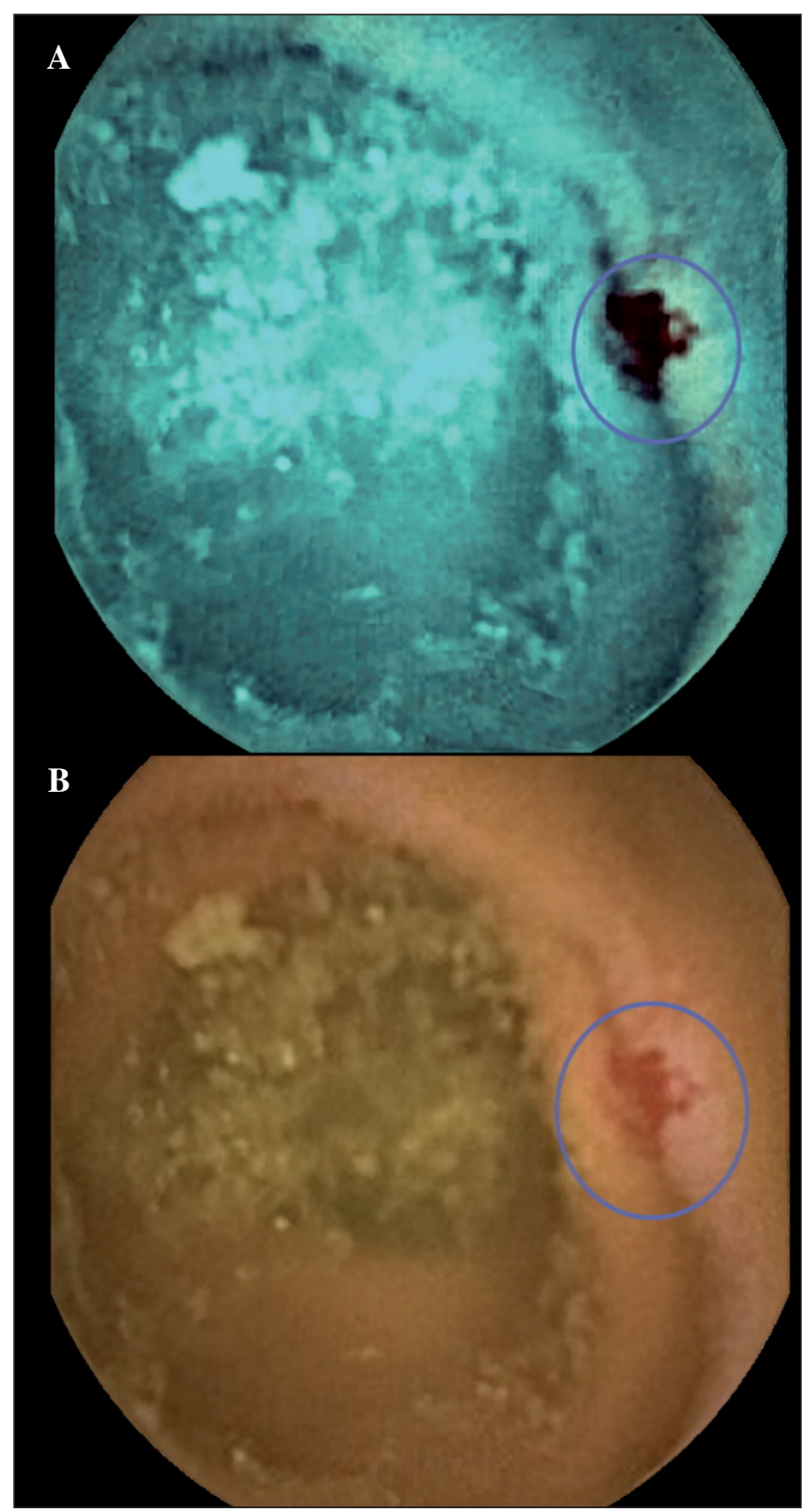

Fig. 4. Angiodysplasia identified with both modes.

Crohn's disease (CD) remains unclear. It has been difficult to correlate the findings in $\mathrm{CE}$ with clinical presentation. The presence of aphthae, erosions or ulcers are indicative of CD although there is no consensus in the number of mucosal breaks that should be considered indicative of a diagnosis of CD (12). While it is the presence of more than three ulcerations, in the absence of NSAIDs ingestion that constitutes the most commonly used diagnostic criteria for $\mathrm{CD}$, proposed by Mow et al. (13), in the study by Voderholzer et al. (14) it is the detection of more than ten ulcerations. Our results show that applying FICE to CE might have an impact in the management of patients with suspected CD, namely by increasing the number of erosions detected. 
As stated by Gupta et al. (15), FICE has the potential to more easily characterize vascular lesions. Curiously, in our study, the 3 additional angiodysplasias identified by CEFICE were detected in patients with other vascular lesions and so the additional findings didn't modified the therapeutic management. However, larger studies are deemed necessary to determine if this technique could improve the diagnostic yield of CE for obscure bleeding.

\section{CONCLUSION}

The use of virtual chromoendoscopy with FICE in CE is an easy-to-apply imaging tool that seems to provide major improvements in image quality and surface analysis, increasing the diagnostic accuracy by detecting erosions and angiodysplasias undetected by the conventional white light mode. The particular population included in this study may anticipate the usefulness of FICE in small bowel bleeding. Further studies are needed to confirm if this new tool is really helpful to identify and/or characterize doubtful lesions.

\section{REFERENCES}

1. McGill S, Soetikno R, Kaltenbach T. Image-enhanced endoscopy in practice. Can J Gastoenterol 2009;11:741-5.

2. Buchner AM, Wallace MB. Future expectations in digestive endoscopy: competition with other novel imaging techniques. Best Pract Res Clin Gastroenterol 2008;22:971-87.

3. Pohl J, May A, Rabenstein T, Pech O, Ell C. Computed virtual chromoendoscopy: a new tool for enhancing tissue surface structures. Endoscopy 2007;39: 80-3.
4. Coriat R, Chryssostalis A, Zeitoun JD, Deyra J, Gaudric M, Prat F, et al. Computed virtual chromoendoscopy system (FICE): a new tool for upper endoscopy. Gastroenterol Clin Biol 2008;32:363-9.

5. Meron GD. The development of the swallowable video capsule (M2A). Gastrointest Endosc 2000;52:817-9.

6. Pohl J, Aschmoneit I, Schumann S, Ell C. Computed image modification for enhancement of small-bowel surface structures at video capsule endoscopy. Endoscopy 2010;42:490-2

7. Imagawa H, Oka S, Tanaka S, Noda I, Higashiyama M, Sanomura Y, et al. Improved visibility of lesions of the small intestine via capsule endoscopy with computed virtual chromoendoscopy. Gastrointestinal Endoscopy 2011;73:299-306.

8. Imagawa H, Oka S, Tanaka S, Noda I, Higashiyama M, Sanomura Y, et al. Improved detectability of small-bowel lesions via capsule endoscopy with computed virtual chromoendoscopy: A pilot study. Scand J Gastroenterol 2011;46:1133-7.

9. Ell C. Impact of virtual chromoendoscopy at colonoscopy: the final requiem for conventional histology? Gastrointestinal Endoscopy 2009;69:723-5.

10. Pohl J, Nguyen-Tat M, Pech O, May A, Rabenstein T, Ell C. Computed virtual chromoendoscopy for classification of small colorectal lesions: a prospective comparative study. Am J Gastroenterol 2008;103(3):562-9.

11. Graham DY, Chan FK. Endoscopic ulcers with low-dose aspirin and reality testing. Gastroenterology 2005;128:807.

12. Figueiredo P, Almeida N, Lopes S, Duque G, Freire P, Lérias C, et al. Small-bowel capsule endoscopy in patients with suspected Crohn's disease-diagnostic value and complications. Diagn Ther Endosc 2010; pii: 101284

13. Mow W, Lo S, Targan S, Dubinsky MC, Treyzon L, Abreu-Martin MT, et al. Initial experience with wireless capsule enteroscopy in the diagnosis and management of inflammatory bowel disease. Clin Gastroenterol Hepatol 2004;2:31-40.

14. Voderholzer W., Beinhoelzl, Rogalla P, Murrer S, Schachschal G, Lochs H, et al. Small bowel involvement in Crohn's disease: a prospective comparison of capsule endoscopy and computed tomography enteroclysis. Gut 2005;54:369-73.

15. Gupta T, Ibrahim M, Deviere J, Van Gossum A. Evaluation of Fujinon Intelligent Chromo Endoscopy-assisted capsule endoscopy in patients with obscure gastroenterology bleeding. World J Gastroenterol 2011; 17:4590-5. 